\title{
The monoid of suspensions and loops modulo Bousfield equivalence
}

by

Jeff Strom (Kalamazoo, MI)

\begin{abstract}
The suspension and loop space functors, $\Sigma$ and $\Omega$, operate on the lattice of Bousfield classes of (sufficiently highly connected) topological spaces, and therefore generate a submonoid $\mathcal{L}$ of the complete set of operations on the Bousfield lattice. We determine the structure of $\mathcal{L}$ in terms of a single parameter of homotopy theory which is closely tied to the problem of desuspending weak cellular inequalities.
\end{abstract}

Introduction. A topological space $Y$ is said to be $X$-acyclic if the weak contractibility of the space of pointed maps from $X$ to $Z$ implies the weak contractibility of the maps from $Y$ to $Z$, that is, if the implication

$$
\operatorname{map}_{*}(X, Z) \sim * \Rightarrow \operatorname{map}_{*}(Y, Z) \sim *
$$

holds for all spaces $Z$. This relation is denoted $X<Y$, and is referred to as a weak cellular inequality. Spaces $X$ and $Y$ are Bousfield equivalent if both $X<Y$ and $Y<X$; the Bousfield equivalence class of $X$ is denoted $\langle X\rangle$. Bousfield classes (of spectra) play an important role in stable homotopy theory, and are crucial to the development of unstable analogs of the nilpotence and periodicity results of Devinatz, Hopkins and Smith.

Chachólski has shown [2] that the suspension and loop space functors $\Sigma$ and $\Omega$ respect weak cellular inequalities; that is, if $X<Y$, then also $\Sigma X<$ $\Sigma Y$ and $\Omega X<\Omega Y$, provided $X$ and $Y$ are both path connected. Consequently, if $\langle X\rangle=\langle Y\rangle$, then $\langle\Sigma X\rangle=\langle\Sigma Y\rangle$ and $\langle\Omega X\rangle=\langle\Omega Y\rangle$. It is easily proved using the James reduced product construction that $\langle\Sigma \Omega \Sigma X\rangle=$ $\langle\Sigma X\rangle$ for any space $X$. Additionally, it was observed in the 1950's that a connected space $X$ is an H-space if and only if it is a retract of $\Omega \Sigma X$. This implies that $\langle\Omega \Sigma \Omega X\rangle=\langle\Omega X\rangle$ for any connected space $X$. It is less obvious, however, that for every path connected space $X$,

$$
\left\langle\Sigma^{3} \Omega^{4} \Sigma^{2} \Omega^{3} \Sigma^{5} X\right\rangle=\left\langle\Sigma^{3} X\right\rangle
$$

2000 Mathematics Subject Classification: 55P60, 55P65, 55P99, $20 \mathrm{M} 05$.

Key words and phrases: Bousfield class, suspension, loop space, cellular inequality. 
and, further, that there are arbitrarily highly connected spaces $X$ for which

$$
\left\langle\Sigma^{3} \Omega^{4} \Sigma^{2} \Omega^{3} \Sigma^{5} X\right\rangle \neq\left\langle\Sigma^{4} \Omega^{5} \Sigma^{3} \Omega^{6} \Sigma^{4} \Omega^{2} \Sigma^{5} X\right\rangle .
$$

The purpose of this paper is to develop a systematic framework for analyzing relations of this kind.

Let $\mathcal{F}$ be the free monoid on the symbols $\Sigma$ and $\Omega$, and let it act on topological spaces in the obvious way. Since the loop space and suspension functors respect Bousfield equivalence (of path connected spaces), this action induces an action of $\mathcal{F}$ on Bousfield classes. We define an equivalence relation $\sim$ on $\mathcal{F}$ by saying $W_{1} \sim W_{2}$ if and only if $\left\langle W_{1} X\right\rangle=\left\langle W_{2} X\right\rangle$ for every sufficiently highly connected space $X$ (see $\S 2.1$ for details concerning the connectivity condition). The set $\mathcal{L}$ of $\sim$-equivalence classes inherits a monoid structure and the quotient map $q: \mathcal{F} \rightarrow \mathcal{L}$ is a monoid map. This map $q$ clearly encodes all relations of the kind discussed above. Our object is to give an explicit description of the monoid $\mathcal{L}$ and the map $q$.

To facilitate our study of $\mathcal{L}$, we introduce two functions, $d: \mathcal{F} \rightarrow \mathbb{Z}$ and $c: \mathcal{F} \rightarrow \mathbb{Z}$, called the degree and the connectivity. The degree of $W \in \mathcal{F}$ is the difference between the connectivity of $X$ and that of $W X$ for highly connected spaces $X$; the connectivity of $W$ is defined by setting $c(\Sigma)=0$, $c(\Omega)=-1$ and $c\left(W_{2} W_{1}\right)=\min \left\{c\left(W_{1}\right), c\left(W_{2}\right)+d\left(W_{1}\right)\right\}$. It is not hard to see that if $W_{1} \sim W_{2}$, then $d\left(W_{1}\right)=d\left(W_{2}\right)$. It will follow from our main theorem that $c$ also respects $\sim$.

Our main result explicitly identifies the monoid $\mathcal{L}$ in terms of an as yet undetermined parameter of homotopy theory:

$$
\kappa=\inf \left\{n \mid \Omega^{n} \Omega \Sigma \sim \Omega^{n}\right\},
$$

where we interpret the infimum of the empty set to be $\infty$, as usual. The parameter $\kappa$ is intimately bound up with the problem of desuspending weak cellular inequalities (see Proposition 12). We conjecture that $\kappa=\infty$.

TheOREM 11. $\mathcal{L}$ is the quotient of $\mathcal{F}$ by $\sim$, which is the unique multiplicative equivalence relation on $\mathcal{F}$ satisfying the following six conditions:

(R1) $\Omega \Sigma^{2} \sim \Sigma$,

(R2) $\Omega \Sigma \Omega \sim \Omega$,

(R3) $\Sigma \Omega \Sigma \sim \Sigma$,

$\left(\mathrm{S}_{\kappa}\right) \Omega^{\kappa} \Omega \Sigma \sim \Omega^{\kappa}$ and $\Omega^{l} \Omega \Sigma \nsim \Omega^{l}$ for all $l<\kappa$,

(N) $\Sigma \Omega^{2} \nsim \Omega$,

(D) $W_{1} \sim W_{2}$ implies $d\left(W_{1}\right)=d\left(W_{2}\right)$.

Let $x_{c, d}=\Sigma^{d-c} \Omega^{-c}$ and $y_{c, d}=\Sigma^{d-c} \Omega^{-c} \Omega \Sigma$; these elements have connectivity $c$ and degree $d$. If $c \leq-\kappa$, then $x_{c, d} \sim y_{c, d}$, but otherwise no two of these words are equivalent. Therefore, every word $W \in \mathcal{F}$ is equivalent 
to exactly one element of the set

$$
\mathcal{R}=\left\{x_{0,0}\right\} \cup\left\{x_{c, d} \mid c<0, d \geq c\right\} \cup\left\{y_{c, d} \mid-\kappa<c \leq 0, d \geq c\right\} .
$$

Writing $\bar{x}_{c, d}$ and $\bar{y}_{c, d}$ for the equivalence classes of $x_{c, d}$ and $y_{c, d}$, we have

$$
\mathcal{L}=\left\{\bar{x}_{0,0}, \bar{x}_{c, d}, \bar{y}_{c, d} \mid c, d \text { as in } \mathcal{R} \text { above }\right\} .
$$

Every nontrivial $W \in \mathcal{F}$ has a unique factorization of the form $W=$ $W^{\prime} T$, where $T \in\{\Sigma, \Omega\}$. We call $T$ the initial letter of $W$ because it is the first operation that is applied in evaluating $W X$ for a space $X$. Theorem 11 implies the following criterion for the equivalence of two words.

Proposition 15. Let $W_{1}, W_{2}$ be nonempty words in $\mathcal{F}$. Then $W_{1} \sim W_{2}$ if the following three conditions hold:

(1) the initial letters of $W_{1}$ and $W_{2}$ are the same,

(2) $d\left(W_{1}\right)=d\left(W_{2}\right)$

(3) $c\left(W_{1}\right)=c\left(W_{2}\right)$.

Conversely, if $W_{1} \sim W_{2}$, then conditions (2) and (3) hold; condition (1) also holds if $c\left(W_{1}\right)=c\left(W_{2}\right)>-\kappa$.

If $W_{1} \sim W_{2}$, then $\left\langle W_{1} X\right\rangle=\left\langle W_{2} X\right\rangle$ for all "sufficiently highly connected" spaces $X$. For practical use, it is essential to know exactly how highly connected $X$ must be for this implication to be valid.

Corollary 16. If conditions (1)-(3) of Proposition 15 hold, then $\left\langle W_{1} X\right\rangle$ $=\left\langle W_{2} X\right\rangle$ for all c-connected spaces $X$, where $c=c\left(W_{1}\right)=c\left(W_{2}\right)$.

Proposition 15 and Corollary 16 do not completely settle the question: without knowing the value of $\kappa$, we cannot say whether or not two words with different initial letters are equivalent.

As mentioned above, Theorem 11 is an implicit description of the quotient map $q: \mathcal{F} \rightarrow \mathcal{L}$. The explicit formula is given in the following proposition.

Proposition 18. Let $W \in \mathcal{F}$ be a nonempty word, and write $d=d(W)$ and $c=c(W)$. Then

$$
q(W)= \begin{cases}\bar{x}_{c, d} & \text { if the initial letter of } W \text { is } \Omega, \\ \bar{y}_{c, d} & \text { if the initial letter of } W \text { is } \Sigma,\end{cases}
$$

where $\bar{x}_{c, d}=\bar{y}_{c, d}$ for $c \leq-\kappa$.

We conclude by describing the multiplicative structure of $\mathcal{L}$ explicitly in terms of the elements $\bar{x}_{c, d}$ and $\bar{y}_{c, d}$.

Proposition 20. Let $\bar{z}_{c, d}$ denote a generic element of $\mathcal{L}$ with the indicated connectivity and degree. The product in $\mathcal{L}$ is given by the formulas 


$$
\bar{z}_{c, d} \cdot \bar{x}_{c^{\prime}, d^{\prime}}=\bar{x}_{m, d+d^{\prime}} \quad \text { and } \quad \bar{z}_{c, d} \cdot \bar{y}_{c^{\prime}, d^{\prime}}=\bar{y}_{m, d+d^{\prime}}
$$

where $m=\min \left\{c^{\prime}, d^{\prime}+c\right\}$ and $\bar{x}_{c, d}=\bar{y}_{c, d}$ for $c \leq-\kappa$.

It follows that the $\bar{x}$-elements constitute a submonoid $X \subseteq \mathcal{L}$ and similarly for the $\bar{y}$-elements.

Acknowledgments. I am pleased to acknowledge the helpful advice provided by W. Chachólski, E. Dror Farjoun, M. Intermont, Lê Minh Hà and M. Kinyon during the preparation of this paper.

1. Preliminaries. An equivalence relation $\approx$ on a monoid $\mathcal{M}$ is multiplicative if $x_{1} \approx x_{1}^{\prime}$ and $x_{2} \approx x_{2}^{\prime}$ implies $x_{1} x_{2} \approx x_{1}^{\prime} x_{2}^{\prime}$. If $\approx$ is a multiplicative equivalence relation, then the set $\mathcal{N}$ of $\approx$-equivalence classes inherits a monoid structure and the quotient $\mathcal{M} \rightarrow \mathcal{N}$ is a monoid map.

An equivalence relation on a set $S$ can be viewed as a subset of $S \times S$ with certain properties. Since an intersection of equivalence relations is also an equivalence relation, any subset $A \subseteq S$ is contained in a unique smallest equivalence relation, the equivalence relation generated by $A$.

Homotopy equivalence of pointed topological spaces is denoted $\simeq$. The loop space of a pointed space $X$ is the pointed space of pointed maps $\Omega X=\operatorname{map}_{*}\left(S^{1}, X\right)$. The Eckman-Hilton dual of the loop space functor is the suspension functor defined by $\Sigma X=S^{1} \wedge X$, where $\wedge$ denotes the smash product. A space $X$ is $n$-connected if $\Omega^{k} X$ is a path connected space for $0 \leq k \leq n ; X$ is weakly contractible if it is $n$-connected for all $n \geq 1$. Refer to [5] for more detail on the basic notions of homotopy theory.

A path connected pointed $\left(^{1}\right)$ space $Y$ is $X$-acyclic if whenever map ${ }_{*}(X, Z)$ is weakly contractible, then $\operatorname{map}_{*}(Y, Z)$ is also weakly contractible. This relation is denoted $X<Y$, and it is a partial order on the collection of topological spaces. Spaces $X$ and $Y$ are Bousfield equivalent if $X<Y$ and $Y<X$. The Bousfield class of $X$ is the class $\langle X\rangle=\{Z \mid Z$ is Bousfield equivalent to $X$ \}. Chachólski proved the following key results in [2, Cors. $18.2 \& 18.4]$.

Proposition 1. If $X$ and $Y$ are path connected and $X<Y$, then $\Sigma X<$ $\Sigma Y$ and $\Omega X<\Omega Y$.

COROllary 2. If $X$ and $Y$ are path connected and $\langle X\rangle=\langle Y\rangle$, then $\langle\Sigma X\rangle=\langle\Sigma Y\rangle$ and $\langle\Omega X\rangle=\langle\Omega Y\rangle$.

2. The framework. In this section we define our object of study: the monoid of operations on the Bousfield lattice generated by the loop space and suspension functors.

$\left({ }^{1}\right)$ In this paper we work exclusively with highly connected spaces, so we do not have to worry about choice of basepoints. 
2.1. The monoid of suspensions and loops. Let $\mathcal{F}$ be the free monoid on the symbols $\Sigma$ and $\Omega$. This monoid acts on pointed topological spaces in the obvious way:

$$
\Sigma \cdot X=\Sigma X \quad \text { and } \quad \Omega \cdot X=\Omega X .
$$

We define an equivalence relation $\sim$ on elements of $\mathcal{F}$ by defining $W_{1} \sim W_{2}$ if and only if there is an integer $n=n\left(W_{1}, W_{2}\right)$ such that $\left\langle W_{1} X\right\rangle=\left\langle W_{2} X\right\rangle$ whenever $X$ is $n$-connected. We refer to $n$-connected spaces as "sufficiently highly connected".

We write $\mathcal{L}$ for the set of $\sim$-equivalence classes of $\mathcal{F}$, and $q: \mathcal{F} \rightarrow \mathcal{L}$ for the canonical map.

Proposition 3. The equivalence relation $\sim$ is multiplicative. Therefore, the set $\mathcal{L}$ inherits a monoid structure from $\mathcal{F}$ for which the canonical map $q: \mathcal{F} \rightarrow \mathcal{L}$ is a monoid map.

Proof. Using Corollary 2, it follows by induction on word length in $\mathcal{F}$ that if $\langle X\rangle=\langle Y\rangle$, then $\langle W X\rangle=\langle W Y\rangle$ for any $W \in \mathcal{F}$ and any sufficiently highly connected spaces $X$ and $Y$. Now suppose that $W_{1} \sim$ $W_{1}^{\prime}$ and $W_{2} \sim W_{2}^{\prime}$. Then, for any sufficiently highly connected space $X$, $\left\langle W_{1} X\right\rangle=\left\langle W_{1}^{\prime} X\right\rangle$ and hence $\left\langle W_{2} W_{1} X\right\rangle=\left\langle W_{2} W_{1}^{\prime} X\right\rangle=\left\langle W_{2}^{\prime} W_{1}^{\prime} X\right\rangle$. Therefore $W_{2} W_{1} \sim W_{2}^{\prime} W_{1}^{\prime}$.

Comment on connectivity. The statement in Proposition 1 about looping weak cellular inequalities is false without the assumption that the spaces in question are connected. For example, consider disjoint unions of Moore spaces $X=M(\mathbb{Z} / p, n) \amalg M(\mathbb{Z} / p, n)$ and $Y=M(\mathbb{Z} / q, n) \amalg M(\mathbb{Z} / q, n)$, where $n$ is large, and $p$ and $q$ are distinct primes. Then $\langle X\rangle=\left\langle S^{0}\right\rangle=\langle Y\rangle$, but $\langle\Omega X\rangle=\langle\Omega M(\mathbb{Z} / p, n)\rangle \neq\langle\Omega M(\mathbb{Z} / q, n)\rangle=\langle\Omega Y\rangle$. Examples of this kind force us to impose the connectivity restiction in the definition of $\sim$; otherwise we would have $\Omega \nsim \Omega$, and $\sim$ would not be an equivalence relation.

2.2. Connectivity and degree. We will make use of two functions on $\mathcal{F}$, $d: \mathcal{F} \rightarrow \mathbb{Z}$ and $c: \mathcal{F} \rightarrow \mathbb{Z}$, called the degree and the connectivity. The function $d$ is determined by the rules

$$
d(\Sigma)=+1, \quad d(\Omega)=-1, \quad d\left(W_{2} W_{1}\right)=d\left(W_{2}\right)+d\left(W_{1}\right) .
$$

If $W \in \mathcal{F}$ is a nontrivial word, write it in the form $W=T_{r} T_{r-1} \cdots T_{2} T_{1}$ where each $T_{k}$ is in $\{\Sigma, \Omega\}$; for $1 \leq k \leq r$, set $W^{(k)}=T_{k} T_{k-1} \cdots T_{2} T_{1}$. Then $c$ is the function defined by setting $c(1)=0$ and

$$
c(W)=\min \left\{d\left(W^{(k)}\right) \mid 1 \leq k \leq r\right\}
$$

for nontrivial $W$. The connectivity $c$ clearly satisfies the equation

$$
c\left(W_{2} W_{1}\right)=\min \left\{c\left(W_{1}\right), d\left(W_{1}\right)+c\left(W_{2}\right)\right\} .
$$


Lemma 4. Let $X$ be a space that is a-connected but not $(a+1)$-connected, and let $W \in \mathcal{F}$ with $d=d(W)$ and $c=c(W)$. If $a+c \geq 0$, then

(a) $W X$ is $(a+d)$-connected,

(b) $W^{(k)} X$ is path connected for all $k$,

(c) if $a \geq 1$, then $W X$ is not $(a+d+1)$-connected,

(d) if $W_{1} \sim W_{2}$, then $d\left(W_{1}\right)=d\left(W_{2}\right)$.

Proof. Part (a) follows by induction because it is true for $\Sigma$ and $\Omega$. Part (a) implies (b) because $c \leq d\left(W^{(k)}\right)$. Part (c) can be proved by tracking the bottom homotopy/homology group using the Hurewicz theorem. For (d), let $W_{1} \sim W_{2}$ with degrees $d_{1}$ and $d_{2}$. Then we can find an $a$-connected space $X$ with $a+c \geq 0$ for which $\left\langle W_{1} X\right\rangle=\left\langle W_{2} X\right\rangle$. This forces the connectivities of $W_{1} X$ and $W_{2} X$ to be equal. By (c), we have $d_{1}+a=d_{2}+a$, so $d_{1}=d_{2}$.

3. Relations in $\mathcal{F}$. In this section we prove the three basic relations between elements of $\mathcal{F}$, and use them to derive other relations. We finish the section by establishing the invalidity of a key relation.

3.1. Three basic relations and some consequences. We begin with our three fundamental relations. We derive some more generally useful relations from them in a corollary afterward.

Proposition 5. For any path connected space $X$,

(a) $\left\langle\Omega \Sigma^{2} X\right\rangle=\langle\Sigma X\rangle$,

(b) $\langle\Omega \Sigma \Omega X\rangle=\langle\Omega X\rangle$,

(c) $\langle\Sigma \Omega \Sigma X\rangle=\langle\Sigma X\rangle$.

Proof. The relation (a) can be traced back as far as Dror Farjoun and Chachólski, but it does not seem to be equivalent to a classical result. First of all, [1, Cor. 3.5] (or the James construction) immediately yields $\Sigma X<$ $\Omega \Sigma(\Sigma X)$. The main point is the reverse inequality. For this, let $F$ be the fiber of the canonical map $\Sigma X \rightarrow \Omega \Sigma(\Sigma X)$, so $F \rightarrow \Sigma X \rightarrow \Omega \Sigma(\Sigma X)$ is a fibration sequence. Then according to [1, Lem. 8.9], $\Omega \Sigma(\Sigma X)<F$, so $\Omega \Sigma(\Sigma X)<\Sigma X$. (Alternatively, combine [3, Cor. 5.B.6] with [1, Thm. 8.4].)

According to [1, Cor. 3.5(3)], $\langle\Omega \Sigma \Omega X\rangle=\langle\Omega X\rangle$ for any space $X$, proving (b). Putting together parts (2) and (4) of [1, Prop. 10.6] yields (c).

Proposition 5 immediately yields the following relations in $\mathcal{F}$.

THEOREM 6 . The following relations are valid in $\mathcal{F}$ :

(R1) $\Omega \Sigma^{2} \sim \Sigma$,

(R2) $\Omega \Sigma \Omega \sim \Omega$,

(R3) $\Sigma \Omega \Sigma \sim \Sigma$.

We will refer to (R1)-(R3) as the three basic relations. They imply the following more generally applicable relations. 
COROLlaRY 7 . The following relations are valid in $\mathcal{F}$ :

(a) $\Omega^{n} \Sigma^{n} \sim \Omega \Sigma$ for $n \geq 1$,

(b) $\left(\Omega^{n} \Sigma^{n}\right) \Sigma \sim \Sigma$ for $n \geq 0$,

(c) $\left(\Omega^{n} \Sigma^{n}\right) \Omega \sim \Omega$ for $n \geq 0$,

(d) $\Sigma\left(\Omega^{n} \Sigma^{n}\right) \sim \Sigma$ for $n \geq 0$.

Proof. Part (a) is the key. To prove it, we proceed by induction on $n$, the case $n=1$ being trivial:

$$
\begin{array}{rlr}
\Omega^{n} \Sigma^{n} & \sim \Omega^{n-1}\left(\Omega \Sigma^{2}\right) \Sigma^{n-2} \\
& \sim \Omega^{n-1} \Sigma \Sigma^{n-2} \quad \text { by }(\mathrm{R} 1) \\
& \sim \Omega^{n-1} \Sigma^{n-1} & \\
& \sim \Omega \Sigma & \text { by the inductive hypothesis. }
\end{array}
$$

Now we use (a) to derive (b)-(d) by directly computing

$$
\begin{array}{ll}
\left(\Omega^{n} \Sigma^{n}\right) \Sigma \sim \Omega \Sigma \Sigma \sim \Sigma & \text { by }(\mathrm{R} 1), \\
\left(\Omega^{n} \Sigma^{n}\right) \Omega \sim \Omega \Sigma \Omega \sim \Omega & \text { by (R2), } \\
\Sigma\left(\Omega^{n} \Sigma^{n}\right) \sim \Sigma \Omega \Sigma \sim \Sigma & \text { by (R3). }
\end{array}
$$

Parts (b) and (c) of Corollary 7 imply the following left cancellation rule for suspensions.

COROllary 8. If $W_{1}, W_{2} \in \mathcal{F}$ are nontrivial words, then $\Sigma^{n} W_{1} \sim$ $\Sigma^{n} W_{2}$ if and only if $W_{1} \sim W_{2}$.

The nontriviality of $W_{1}$ and $W_{2}$ is an essential hypothesis in Corollary 8.

3.2. An irrelation. We will use the following irrelation to clarify the structure of the monoid $\mathcal{L}$.

Proposition 9. $\Sigma \Omega^{k+1} \nsim \Omega^{k}$ for $k \geq 0$.

Proof. Fix an odd prime $p$, and let $K_{n}=K(\mathbb{Z} / p, n)$. Then, according to Ravenel and Wilson [4], $K_{n+1}$ is acyclic with respect to the $p$-local Morava K-theory $K(n)_{*}$ and $K_{n}$ is not $K(n)_{*}$-acyclic. It follows that $K_{n+1} \nless \Sigma K_{n}$ for all $n \geq 1$. Since we have canonical equivalences $K_{n+1} \simeq \Omega^{k} K_{n+k+1}$ and $K_{n} \simeq \Omega^{k+1} K_{n+k+1}$,

$$
\Omega^{k} K_{n+k+1} \simeq K_{n+1} \nless \Sigma K_{n} \simeq \Sigma \Omega^{k+1} K_{n+k+1} .
$$

These spaces can be chosen to be arbitrarily highly connected by adjusting $n$, so the result is proved.

4. The main theorem. Our main result describes the monoid $\mathcal{L}$ and the quotient map $q: \mathcal{F} \rightarrow \mathcal{L}$. It turns out that the structure of $\mathcal{L}$ and the map $q$ are purely algebraic consequences of the information established in Sections 2 and 3. 
4.1. The monoids $\mathcal{M}_{k}$. Our object in this subsection is a purely algebraic investigation of multiplicative equivalence relations $\approx$ on $\mathcal{F}$ that have the following basic properties:

(R1) $\Omega \Sigma^{2} \approx \Sigma$,

(R2) $\Omega \Sigma \Omega \approx \Omega$,

(R3) $\Sigma \Omega \Sigma \approx \Sigma$,

$\left(\mathrm{S}_{k}\right) \Omega^{k} \Omega \Sigma \approx \Omega^{k}$ and $\Omega^{l} \Omega \Sigma \not \approx \Omega^{l}$ for all $l<k$,

(N) $\Sigma \Omega^{2} \not \approx \Omega$,

(D) $W_{1} \approx W_{2}$ implies $d\left(W_{1}\right)=d\left(W_{2}\right)$.

We interpret $\left(\mathrm{S}_{\infty}\right)$ to be the empty relation. Let $\sim_{k}$ be the multiplicative equivalence relation on $\mathcal{F}$ generated by $(\mathrm{R} 1)-(\mathrm{R} 3),\left(\mathrm{S}_{k}\right),(\mathrm{N})$ and $(\mathrm{D})$; let $\mathcal{M}_{k}$ be the monoid of $\sim_{k}$-equivalence classes in $\mathcal{F}$.

Comment on $\left(\mathrm{S}_{k}\right)$. The condition $\Omega^{k} \Omega \Sigma \approx \Omega^{k}$ implies $\Omega^{k+1} \Omega \Sigma \approx$ $\Omega^{k+1}$, so $W_{1} \sim_{k} W_{2}$ implies $W_{1} \sim_{l} W_{2}$ for $l \leq k$. It seems likely that $\Omega^{k} \Omega \Sigma \approx \Omega^{k}$ does not imply $\Omega^{k-1} \Omega \Sigma \approx \Omega^{k-1}$ for any $k$, but we have not proved this. Thus we have not ruled out the possibility that $\left(\mathrm{S}_{k}\right)$ might be inconsistent with (R1)-(R3), (N) and (D) for certain values of $k$.

Proposition 10. Let $\approx$ be any multiplicative equivalence relation which satisfies $(\mathrm{R} 1)-(\mathrm{R} 3),\left(\mathrm{S}_{k}\right),(\mathrm{N})$, and $(\mathrm{D})$. Then

(a) the equivalence relations $\approx$ and $\sim_{k}$ coincide;

(b) every element $W \in \mathcal{F}$ is $\sim_{\infty}$-equivalent to one of the following standard forms:

$$
\begin{array}{ll}
x_{c, d}=\Sigma^{d-c} \Omega^{-c} & \text { for } d \geq c \text { and } c<0, \\
y_{c, d}=\Sigma^{d-c} \Omega^{-c}(\Omega \Sigma) & \text { for } d \geq c \text { and } c \leq 0, \\
x_{0,0}=1 &
\end{array}
$$

(note that $c=c\left(x_{c, d}\right)=c\left(y_{c, d}\right)$ and $\left.d=d\left(x_{c, d}\right)=d\left(y_{c, d}\right)\right)$;

(c) if $W \in \mathcal{F}$ with $c=c(W)$ and $d=d(W)$, then

(i) if $c \leq-k$, then $W \approx x_{c, d} \approx y_{c, d}$ only,

(ii) if $c>-k$, then $W$ is $\approx$-equivalent to $x_{c, d}$ or $y_{c, d}$, but not both.

Proof. We prove (a) as a consequence of (b) and (c) as follows. The conclusion (b) for $\sim_{\infty}$ implies that (b) holds for both $\approx$ and $\sim_{k}$. Also, (c) holds for both $\approx$ and $\sim_{k}$. Let

$$
\mathcal{R}=\left\{x_{0,0}\right\} \cup\left\{x_{c, d} \mid d \geq c, c<0\right\} \cup\left\{y_{c, d} \mid d \geq c,-k<c \leq 0\right\} .
$$

Then (b) and (c) together imply that every word $W \in \mathcal{F}$ is $\approx$-equivalent to exactly one element of $\mathcal{R}$, and similarly for $\sim_{k}$. Clearly, $W_{1} \sim_{k} W_{2}$ implies $W_{1} \approx W_{2}$; we have to prove the reverse implication. Suppose $W_{1} \approx W_{2}$, and let $z_{1}, z_{2} \in \mathcal{R}$ be the unique elements such that $W_{1} \sim_{k} z_{1}$ and $W_{2} \sim_{k} z_{2}$. Then we have $z_{1} \approx W_{1} \approx W_{2} \approx z_{2}$. Since no two distinct elements in the 
set $\mathcal{R}$ are $\approx$-equivalent by (c), it must be that $z_{1}=z_{2}$, and so $W_{1} \sim_{k} z_{1}=$ $z_{2} \sim_{k} W_{2}$, which completes the proof of (a).

Now we prove (b). By repeated use of (R1) (or, equivalently, by Corollary 7), each word $W \in \mathcal{F}$ is $\sim_{\infty}$-equivalent to one in which no $\Omega$ appears to the left of a $\Sigma^{2}$. Thus $W$ is $\sim_{\infty}$-equivalent to a word of the form

$$
\Sigma^{a} \Omega^{b_{1}} \Sigma \Omega^{b_{2}} \Sigma \Omega^{b_{3}} \cdots \Omega^{b_{r-1}} \Sigma \Omega^{b_{r}} \Sigma^{\varepsilon}
$$

where $\varepsilon=0$ or $\varepsilon=1$ and each $b_{i}>0$. Using (R2), we remove each internal $\Sigma$, showing that $W \sim_{\infty} \Sigma^{a} \Omega^{b} \Sigma^{\varepsilon}$ for some $a$ and $b$. Finally, if $a \geq \varepsilon=b=1$, then (R3) reduces the word further, showing that $W \sim_{\infty} \Sigma^{a}$.

To prove (c), we show that no two distinct elements of the set $\mathcal{R}$ are $\approx$-equivalent to one another. By property (D), the only possiblities for such relations are

$x_{c, d} \approx y_{c^{\prime}, d} \quad$ for any $c, c^{\prime}, \quad x_{c, d} \approx x_{c^{\prime}, d} \quad$ for $c \neq c^{\prime}, \quad y_{c, d} \approx y_{c^{\prime}, d} \quad$ for $c \neq c^{\prime}$.

First consider the possibility that $x_{c, d} \approx y_{c, d}$ (note that if $c=0$, then $d=0$ as well). By Corollary 8 we can cancel $\Sigma^{d-c}$ on the left, so the relations

$$
\Sigma^{d-c} \Omega^{-c} \approx \Sigma^{d-c} \Omega^{-c} \Omega \Sigma, \quad \Omega^{-c} \approx \Omega^{-c} \Omega \Sigma,
$$

are equivalent to one another. Since the latter relation is valid precisely when $c \leq-k$ by $\left(\mathrm{S}_{k}\right), x_{c, d} \approx y_{c, d}$ if and only if $c \leq-k$.

We now show that any other relation within the list of part (b) implies the (false) relation $\Sigma \Omega^{2} \approx \Omega$. In fact, if $c \neq c^{\prime}$, then

$$
\begin{aligned}
x_{c, d} & \approx y_{c^{\prime}, d}, \\
\Sigma^{d-c} \Omega^{-c} & \approx \Sigma^{d-c^{\prime}} \Omega^{-c^{\prime}} \Omega \Sigma, \\
\left(\Sigma^{d-c} \Omega^{-c}\right) \Omega & \approx\left(\Omega^{d-c^{\prime}} \Sigma^{d-c^{\prime}} \Omega^{-c^{\prime}} \Omega \Sigma\right) \Omega, \\
\Sigma^{d-c} \Omega^{-c+1} & \approx \Sigma^{d-c^{\prime}} \Omega^{-c^{\prime}+1} \Omega \quad \text { by }(\mathrm{R} 2), \\
x_{c-1, d-1} & \approx x_{c^{\prime}-1, d-1} .
\end{aligned}
$$

In the same way, if $y_{c, d} \approx y_{c^{\prime}, d}\left(\right.$ with $\left.c \neq c^{\prime}\right)$, then $x_{c-1, d-1} \approx x_{c^{\prime}-1, d-1}$, and so it suffices to show that the equivalence of any two of the $x$ terms implies the (invalid) relation $\Sigma \Omega^{2} \approx \Omega$. Assume that $c<c^{\prime}$, which implies $d-c>d-c^{\prime}$, and

$$
\begin{array}{rlr}
x_{c+2, d+2} & \approx x_{c^{\prime}+2, d+2}, & \\
\Sigma^{d-c} \Omega^{-c-2} & \approx \Sigma^{d-c^{\prime}} \Omega^{-c^{\prime}-2}, & \\
\Sigma^{d-c} \Omega^{-c} & \approx \Sigma^{d-c^{\prime}} \Omega^{-c^{\prime}} & \\
\Omega^{d-c-1}\left(\Sigma^{d-c} \Omega^{-c}\right) & \approx \Omega^{d-c-1}\left(\Sigma^{d-c^{\prime}} \Omega^{-c^{\prime}}\right), &
\end{array}
$$




$$
\begin{aligned}
\Sigma \Omega^{-c} & \approx \Omega^{-c-1} & & \text { by Corollary } 7(\mathrm{~b}),(\mathrm{c}) \\
\left(\Sigma \Omega \Omega^{-c-1}\right) \Sigma^{-c-1} & \approx\left(\Omega^{-c-1}\right) \Sigma^{-c-1}, & & \\
\Sigma \Omega^{2} \Sigma & \approx \Omega \Sigma & & \text { by Corollary } 7(\mathrm{a}),(\mathrm{b}), \\
\left(\Sigma \Omega^{2} \Sigma\right) \Omega & \approx(\Omega \Sigma) \Omega, & & \\
\Sigma \Omega^{2} & \approx \Omega & & \text { by }(\mathrm{R} 2) .
\end{aligned}
$$

By our assumption $(\mathrm{N})$, this last relation is not valid, so we have proved that each $W \in \mathcal{F}$ is $\approx$-equivalent to exactly one element of $\mathcal{R}$.

It remains to determine which elements in $\mathcal{R}$ could possibly represent a given $W \in \mathcal{F}$. Since each of the relations $(\mathrm{R} 1)-(\mathrm{R} 3)$ and $\left(\mathrm{S}_{k}\right)$ preserves connectivity, if $W_{1} \approx W_{2}$ then $c\left(W_{1}\right)=c\left(W_{2}\right)$ and $d\left(W_{1}\right)=d\left(W_{2}\right)$. Writing $c(W)=c$ and $d(W)=d$, we conclude that $W$ can only be equivalent to $x_{c, d}$ or $y_{c, d}$, and the proof of (c) is complete.

4.2. The structure of $\mathcal{L}$. We determine the structure of $\mathcal{L}$ in terms of a new and as yet undetermined parameter of homotopy theory: $\kappa=\inf \{n \mid$ $\left.\Omega^{n} \Omega \Sigma \sim \Omega^{n}\right\}$.

\section{TheOREM 11.}

(a) $\mathcal{L} \cong \mathcal{M}_{\kappa}$,

(b) writing $\bar{x}_{c, d}$ and $\bar{y}_{c, d}$ for the $\sim$-equivalence classes of $x_{c, d}$ and $y_{c, d}$, the map $q: \mathcal{F} \rightarrow \mathcal{L}$ is determined by $q(\Sigma)=\bar{y}_{0,1}$ and $q(\Omega)=\bar{x}_{-1,-1}$.

Proof. We know from Proposition 3 that $\sim$ is a multiplicative equivalence relation. It follows from Theorem 6 that $(\mathrm{R} 1)-(\mathrm{R} 3)$ are valid for $\sim$, and $\left(\mathrm{S}_{\kappa}\right)$ holds by definition. Since $(\mathrm{N})$ holds by Proposition 9 and $(\mathrm{D})$ is Lemma 4(d), part (a) follows from Proposition 10. Part (b) is immediate.

The number $\kappa$ is a new and fundamental parameter of homotopy theory. It is intimately related to the problem of desuspending weak cellular inequalities, as the next propostion shows.

Proposition 12. The following are equivalent:

(a) $\Omega^{k} \Omega \Sigma \sim \Omega^{k}$

(b) for sufficiently highly connected spaces $A$ and $X$,

$$
\Sigma\left(\Sigma^{k} A\right)<\Sigma X \Leftrightarrow \Sigma^{k} A<X .
$$

The proof is a straightforward generalization of the proof of Chachólski's desuspension theorem [1, Thm. 8.4]. We omit the details.

Conjecture. $\kappa=\infty$. 
5. Consequences. The purpose of this section is to detail certain consequences of Theorem 11 . We begin by showing that $c$-connected spaces are "sufficiently highly connected" to conclude $\left\langle W_{1} X\right\rangle=\left\langle W_{2} X\right\rangle$ from $W_{1} \sim_{\infty}$ $W_{2}$, where $c=c\left(W_{1}\right)=c\left(W_{2}\right)$. We give an easily applied criterion for deciding when two words are $\sim$-equivalent, and derive an explicit formula for the quotient map $q$. Finally, we give an explicit description of the multiplicative structure of $\mathcal{L}$.

5.1. Clarification on connectivity. If $W_{1} \sim W_{2}$, then $\left\langle W_{1} X\right\rangle=\left\langle W_{2} X\right\rangle$ for all "sufficiently highly connected" spaces $X$. For practical use, it is essential to know exactly how highly connected $X$ must be for this implication to be valid.

Proposition 13.

(a) If $W_{1} \sim W_{2}$, then $c\left(W_{1}\right)=c\left(W_{2}\right)$.

(b) If $W_{1} \sim_{\infty} W_{2}$ with $c=c\left(W_{1}\right)=c\left(W_{2}\right)$, then $\left\langle W_{1} X\right\rangle=\left\langle W_{2} X\right\rangle$ for all c-connected spaces $X$.

Proof. Part (a) follows from Proposition 10(c), because $\sim$ and $\sim_{\kappa}$ are the same equivalence relation by Theorem 11 .

If $W_{1} \sim_{\infty} W_{2}$, then $W_{1}$ can be transformed into $W_{2}$ by a finite sequence of substitutions made using the three basic relations. Our claim will follow by induction, once we verify it for the case in which $W_{1}$ and $W_{2}$ differ by exactly one such substitution. Write $W_{1}=U L V$ and $W_{2}=U R V$, where $L \sim R$ is one of the three basic relations (in any order).

If $X$ is c-connected, then $W_{1}^{(k)} X$ and $W_{2}^{(k)} X$ are path connected for all $k$. In particular, $V X$ is path connected, so $\langle L V X\rangle=\langle R V X\rangle$ by Proposition 5. Also, $U^{(k)} L V X$ and $U^{(k)} R V X$ are path connected, and it follows by induction on $k$ that $\left\langle U^{(k)} L V X\right\rangle=\left\langle U^{(k)} R V X\right\rangle$ for all $k \geq 0$, proving (b).

We are unable to prove $(b)$ for the relation $\sim$ because we do not know enough about $\kappa$. If $\kappa=\infty$, then the proof given above applies directly to $\sim$. But if $\kappa<\infty$, then there are relations of the form $\Omega^{k} \Omega \Sigma \sim \Omega^{k}$; and we do not know how highly connected a space must be in order to render such a relation valid.

5.2. Determining equivalences. Next we address our motivating problem by giving a simple criterion for deciding whether two words in $\mathcal{F}$ are equivalent.

We begin with a lemma. It is generally true that $W_{1} \sim_{\infty} W_{2}$ implies $W_{1} \sim W_{2}$; we prove that the converse is true for words of comparatively high connectivity.

LEMMA 14. If $c=c\left(W_{1}\right)=c\left(W_{2}\right)>-\kappa$, then $W_{1} \sim W_{2}$ if and only if $W_{1} \sim_{\infty} W_{2}$. 
Proof. Suppose $W_{1} \sim W_{2}$ and let $d=d\left(W_{1}\right)=d\left(W_{2}\right)$. By Proposition 10 , there are representatives $z_{1}, z_{2} \in \mathcal{R}$ such that $W_{1} \sim_{\infty} z_{1}$ and $W_{2} \sim_{\infty} z_{2}$. Therefore $z_{1} \sim W_{1} \sim W_{2} \sim z_{2}$. Furthermore, $z_{1}, z_{2} \in\left\{x_{c, d}, y_{c, d}\right\}$ because degree and connectivity respect $\sim$. We know that $c>-\kappa$, so $x_{c, d} \nsim y_{c, d}$, and hence $z_{1}=z_{2}$. Therefore $W_{1} \sim_{\infty} z_{1}=z_{2} \sim_{\infty} W_{2}$.

If $W \in \mathcal{F}$, we say that the initial letter of $W$ is the unique letter $T \in$ $\{\Sigma, \Omega\}$ such that $W$ can be factored $W=W^{\prime} T$.

Proposition 15. Let $W_{1}, W_{2}$ be nonempty words in $\mathcal{F}$. Then $W_{1} \sim_{\infty}$ $W_{2}$ (and, a fortiori, $W_{1} \sim W_{2}$ ) if the following three conditions hold:

(1) the initial letters of $W_{1}$ and $W_{2}$ are the same,

(2) $d\left(W_{1}\right)=d\left(W_{2}\right)$,

(3) $c\left(W_{1}\right)=c\left(W_{2}\right)$.

Conversely, if $W_{1} \sim W_{2}$, then conditions (2) and (3) hold; condition (1) also holds if $c\left(W_{1}\right)=c\left(W_{2}\right)>-\kappa$.

Proof. Each of the three basic relations (R1)-(R3) preserves initial letters, while $\left(\mathrm{S}_{k}\right)$ does not unless $k=\infty$.

Suppose that the three conditions hold for $W_{1}$ and $W_{2}$. If their common initial letter is $\Omega$, then by Proposition 10 they are each $\sim_{\infty}$-equivalent to some word of the form $\Sigma^{a} \Omega^{b}$. This forces $W_{1} \sim W_{2}$, because there is a unique word of that form with given connectivity and degree. If the common initial letter is a $\Sigma$, then $W_{1}$ and $W_{2}$ must each be $\sim_{\infty}$-equivalent to a word of the form $\Sigma^{a} \Omega^{b} \Omega \Sigma$. Again, there is a unique such word with given connectivity and degree. This completes the proof of the forward implication.

Suppose now that $W_{1} \sim W_{2}$. We have already seen that $c\left(W_{1}\right)=c\left(W_{2}\right)$ and $d\left(W_{1}\right)=d\left(W_{2}\right)$. If $c\left(W_{1}\right)=c\left(W_{2}\right)>-\kappa$, then $W_{1} \sim_{\infty} W_{2}$ by Lemma 14 , so $W_{1}$ and $W_{2}$ must have the same initial letters.

Corollary 16. If $W_{1}$ and $W_{2}$ satisfy conditions (1)-(3) of Proposition 15 , then $\left\langle W_{1} X\right\rangle=\left\langle W_{2} X\right\rangle$ for all c-connected spaces $X$, where $c=$ $c\left(W_{1}\right)=c\left(W_{2}\right)$.

Proposition 15 also implies the following extension of Proposition 13.

Corollary 17. Suppose $W_{1} \sim W_{2}$, where $W_{1}=W_{1}^{\prime} \Sigma$ and $W_{2}=W_{2}^{\prime} \Sigma$ with $c\left(W_{1}^{\prime}\right)=c\left(W_{2}^{\prime}\right)=0$. Then $\left\langle W_{1} X\right\rangle=\left\langle W_{2} X\right\rangle$ for every space $X$.

Proof. Since $\left\langle W_{1} X\right\rangle=\left\langle W_{1}^{\prime}(\Sigma X)\right\rangle$ and $\left\langle W_{2} X\right\rangle=\left\langle W_{2}^{\prime}(\Sigma X)\right\rangle$ and $\Sigma X$ is 0 -connected, it suffices by Proposition $13(\mathrm{~b})$ to show that $W_{1} \sim W_{2}^{\prime}$. Clearly, $d\left(W_{1}^{\prime}\right)=d\left(W_{2}^{\prime}\right)$, and $c\left(W_{1}^{\prime}\right)=c\left(W_{2}^{\prime}\right)$ by hypothesis. The initial letters of $W_{1}^{\prime}$ and $W_{2}^{\prime}$ must be $\Sigma$, because otherwise the connectivity would be negative. Thus, the result follows from Proposition 15. 
5.3. The map $q$. The proof of Proposition 15 contains, in essence, a complete description of the map $q$.

Proposition 18. Let $W \in \mathcal{F}$ be a nonempty word, and write $d=d(W)$ and $c=c(W)$. Then

$$
q(W)= \begin{cases}\bar{x}_{c, d} & \text { if the initial letter of } W \text { is } \Omega \\ \bar{y}_{c, d} & \text { if the initial letter of } W \text { is } \Sigma,\end{cases}
$$

where $\bar{x}_{c, d}=\bar{y}_{c, d}$ for $c \leq-\kappa$.

To illustrate the use of these results, we verify the claims made in the introduction.

EXAMPLE 19. Let $W_{1}=\Sigma^{3} \Omega^{4} \Sigma^{2} \Omega^{3} \Sigma^{5}$ and $W_{2}=\Sigma^{4} \Omega^{5} \Sigma^{3} \Omega^{6} \Sigma^{4} \Omega^{2} \Sigma^{5}$ as in the introduction. Direct computation yields $d\left(W_{1}\right)=3, c\left(W_{1}\right)=0$, $d\left(W_{2}\right)=3$, and $c\left(W_{2}\right)=-1$, so Proposition 18 and Theorem 11 show that

$$
\Sigma^{3} \Omega^{4} \Sigma^{2} \Omega^{3} \Sigma^{5} \sim \Sigma^{3} \nsim \Sigma^{4} \Omega^{2} \Sigma \sim \Sigma^{3} \Omega^{4} \Sigma^{3} \Omega^{6} \Sigma^{4} \Omega^{2} \Sigma^{5} .
$$

Finally, since $c\left(W_{1}\right)=0$ and $\Sigma^{3} \Omega^{4} \Sigma^{2} \Omega^{3} \Sigma^{5} \sim \Sigma^{3}$, Proposition 13(b) implies $\left\langle\Sigma^{3} \Omega^{4} \Sigma^{2} \Omega^{3} \Sigma^{5} X\right\rangle=\left\langle\Sigma^{3} X\right\rangle$ for all path connected spaces $X$.

5.4. The multiplicative structure of $\mathcal{L}$. We describe the multiplication in $\mathcal{L}$ explicitly in terms of the elements $\bar{x}_{c, d}$ and $\bar{y}_{c, d}$.

Proposition 20. Let $\bar{z}_{c, d}$ denote a generic element of $\mathcal{L}$ with the indicated connectivity and degree. The product in $\mathcal{L}$ is given by the formulas

$$
\bar{z}_{c, d} \cdot \bar{x}_{c^{\prime}, d^{\prime}}=\bar{x}_{m, d+d^{\prime}} \quad \text { and } \quad \bar{z}_{c, d} \cdot \bar{y}_{c^{\prime}, d^{\prime}}=\bar{y}_{m, d+d^{\prime}},
$$

where $m=\min \left\{c^{\prime}, d^{\prime}+c\right\}$ and $\bar{x}_{c, d}=\bar{y}_{c, d}$ for $c \leq-\kappa$.

Proof. It follows from the definition of $d$ and from Proposition 13 that

$$
c\left(\bar{z}_{c, d} \cdot \bar{z}_{c^{\prime}, d^{\prime}}\right)=\min \left\{c^{\prime}, d^{\prime}+c\right\}=m \quad \text { and } \quad d\left(\bar{z}_{c, d} \cdot \bar{z}_{c^{\prime}, d^{\prime}}\right)=d+d^{\prime},
$$

so it remains to determine whether the products in question are $x$ 's or $y$ 's. According to Proposition 15, this is determined by the initial letter of the product when $c>-\kappa$, and there is no distinction between $x$ 's and $y$ 's when $c \leq-\kappa$.

\section{Final comments and questions}

Variations. There are two evident variations on the basic framework we have established. First of all, we could use cellular inequalities $\ll$ instead of weak cellular inequalities $<$ to define equivalence of words $W \in \mathcal{F}$. In this case, the relation (R1) fails, because, for example, $\Omega \Sigma^{2} S^{n} \nless \Sigma S^{n}$ for all $n>6[2,20.10]$. Secondly, we could use different test spaces $X$ to decide equivalence. For example, we could test potential equivalences in $\mathcal{F}$ on the $\mathcal{F}$-orbit of (sufficiently highly connected) finite complexes. In this case, our proof of $(\mathrm{N})$ fails. 
A partial order on $\mathcal{L}$. Weak cellular inequalities can be used to define compatible partial orders on the monoids $\mathcal{F}$ and $\mathcal{L}$ by setting $W_{1}<W_{2}$ if and only if $W_{1} X<W_{2} X$ for all sufficiently highly connected spaces $X$. Then $<$ is a partial order on $\mathcal{L}$ that respects multiplication; also, $W_{1}<W_{2}$ if and only if $q\left(W_{1}\right)<q\left(W_{2}\right)$. Clearly, $W_{1} \sim W_{2}$ if and only if $W_{1}<W_{2}$ and $W_{2}<W_{1}$. The methods of this paper can be used to show that

(a) $x_{c, d}<x_{c^{\prime}, d^{\prime}}$ if and only if $d \leq d^{\prime}$ and $c \leq c^{\prime}$,

(b) $x_{c, d}<y_{c^{\prime}, d^{\prime}}$ if and only if $d \leq d^{\prime}$ and $c \leq c^{\prime}$,

(c) $y_{c, d}<y_{c^{\prime}, d^{\prime}}$ if and only if $d \leq d^{\prime}$ and $c \leq c^{\prime}$.

We have been unable to resolve the inequalities of the form $y_{c, d}<x_{c^{\prime}, d^{\prime}}$ (see Question 2 below).

Questions. We conclude with two problems whose solution would complete the description of the structure of $\mathcal{L}$ as a monoid, and as an ordered monoid.

(1) Determine the parameter $\kappa$. We conjecture that $\kappa=\infty$, but it would be very interesting if it were shown to be finite.

(2) More generally, determine the values of $k$ and $l$ for which $\Omega^{k} \Omega \Sigma$ $<\Omega^{l}$.

\section{References}

[1] W. Chachólski, Desuspending and delooping cellular inequalities, Invent. Math. 129 (1997), 37-62.

[2] - On the functors $C W_{A}$ and $P_{A}$, Duke Math. J. 84 (1996), 599-631.

[3] E. Dror Farjoun, Cellular Spaces, Null Spaces and Homotopy Localization, Lecture Notes in Math. 1622, Springer, Berlin, 1996.

[4] D. C. Ravenel and W. S. Wilson, The Morava K-theories of Eilenberg-Mac Lane spaces and the Conner-Floyd conjecture, Amer. J. Math. 102 (1980), 691-748.

[5] G. W. Whitehead, Elements of Homotopy Theory, Grad. Texts in Math. 61, Springer, 1978.

Department of Mathematics

Western Michigan University

1903 W. Michigan Ave.

Kalamazoo, MI 49008, U.S.A.

E-mail: Jeff.Strom@wmich.edu

Received 5 January 2006;

in revised form 27 January 2008 